Doch werden wir versuchen, die Metallverbindungen des Glycols auf anderem Wege zu erhalten; denn die Verbindung mit den Alkalimetallen gäbe ein Mittel an die Hand, durch Einwirkung von $\mathrm{CO}_{2}$ die zweibasische Bernsteinsäure künstlich zu erhalten, nach dem Schema :

$$
\left.\left.\underset{\mathrm{Na}_{2}}{\mathrm{C}_{4}^{\prime \prime} \mathrm{H}_{4}}\right\}+2 \mathrm{C}_{2} \mathrm{O}_{4}=\underset{\mathrm{Na}_{2}}{\mathrm{C}_{8} \mathrm{H}_{4} \mathrm{O}_{4}}\right\} \mathrm{O}_{4}
$$

welche Reaction derjenigen ganz analog wäre, nach welcher einer von uns aus Natriumäthyl und $\mathrm{CO}_{2}$ die Propionsäure*) und später die Essigsäure ${ }^{* *}$ ) künstlich dargestellt hat.

\title{
Zusammensetzung des Gases im dunkelen Kegel nicht leuchtender Gasflammen;
}

$$
\text { von Dr. Georg Lunge. }
$$

Das Gasgemenge, welches im dunkelen Kegel der Flamme des von Professor Bunsen angegebenen Gasbrenners enthalten ist, besteht aus einem Gemisch von atmosphärischer Luft und Leuchtgas. Da es von Interesse zu sein schien, zu ermitteln, in welchen Verhältnissen diese beiden Gemengtheile vorhanden seien, so unternahm ich auf Veranlassung des Herrn Hofrath Bunsen eine Analyse des erwähnten Gasgemenges.

Um dasselbe in der zur Analyse nöthigen Menge zu erhalten, wandte ich folgendes Verfahren an : In den oberen Theil des Brenners war einige Millimeter unter der Mündung

\footnotetext{
*) Diese Annalen CVII, 125.

**) Dasclbst CXI, 234 .
} 
eine Oeffnung gebohrt, durch welche eine ziemlich fein ausgezogene, gebogene Glasröhre in der Art geführt war, dals sie genau in der Mitte der Mündung des Brenners senkrecht stand und noch $17^{\mathrm{mm}}$ über dieselbe hervorragte; der Durchmesser ihrer oberen Oeffnung betrug ungefähr $0,3 \mathrm{~mm}$. Diese Glasröhre war in dem Bohrloch des Brenners vollkommen luftdicht eingegypst und ihr anderes Ende durch ein Caoulchoucröhrchen mit zwei an den Enden ausgezogenen weiten Glasröhren verbunden, wie sie gewöhnlich zum Aufsammeln von Gasen benulzt werden. Die lelzte dieser Röhren führte zu einem Aspirator mit constantem Druck, dessen Abflufs durch einen Hahn beliebig geregelt werden konnte. Alle Caoutchoucverbindungen waren mit Schrauben-Quetschhähnen versehen.

Dem Brenner wurde nun, nachdem auf seinen Stern ein conischer Schornstein gesetzt war, welcher noch $47,8^{\text {ma }}$ über die Mündung hervorragte, eine Flamme gegeben, die im Ganzen ungefähr $136^{\mathrm{mm}}$ hoch war; der innere dunkele Kegel hatte eine Höhe von ungefähr $61^{\mathrm{mm}}$. Aus dieser Flamme wurde nun durch die kleine Glasröhre, welche $17^{\mathrm{mm}}$ in sie hineinragte, ein langsamer, die Dimensionen der Flamme kaum merklich ändernder Gasstrom gesaugt, dann der Aspiratorhahn und zugleich sämmtliche Quetschhähne geschlossen, so dafs in den beiden Auffangungsröhren gesonderte Portionen des Gases enthalten waren. Unmittelbar darauf wurden diese unter Quecksilber gebracht und das Gas in ein Absorptionsrohr eingefült. Zur Absorption der Kohlensäure wurde nun eine Kalikugel eingeführt und $\mathbf{1 2}$ Stunden darin gelassen, und darauf zur Absorption des Sauerstoffes eine mit Lösung von pyrogallussaurem Kali getränkte Papiermachékugel 24 Stunden lang mit dem Gase in Berührung gebracht. Die Ablesungen waren : 


\begin{tabular}{|c|c|c|c|c|c|}
\hline & & Corr. Vol. & Druck & Temp. ${ }^{\circ} \mathrm{C}$. & $\begin{array}{l}\text { Vol b. } 0^{0} \\
\text { u. } 1^{\mathbf{m}} \mathrm{Dr} .\end{array}$ \\
\hline Anfängl. & Volum & 144,7 & 0,7024 & 28,1 & 92,15 \\
\hline- & Kohlensäure & 141,4 & 0,7247 & 28,3 & 92,83 \\
\hline - & Sauerstoff & 122,9 & 0,7078 & 27,6 & 79,00 \\
\hline
\end{tabular}

Dieses ergiebt, auf 100 Theile berechnet :

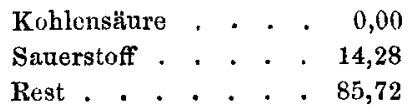

Jetzt wurde ein Theil des Gasrückstandes in ein grofses Eudiometer eingeführt; zu diesem Theile wurde Sauerstoff zugeselzt, explodirt, die Kohlensäure durch eine Kalikugel entfernl, Wasserstoffgas zugesetzt und wieder explodirt. Die Ablesungen waren :

\begin{tabular}{|c|c|c|c|c|}
\hline & Corr. Vol. & Druck & Temp. ${ }^{\circ} \mathrm{C}$. & $\begin{array}{l}\text { Vol. b. } 0^{0} \\
\text { u. } 1^{\mathrm{m}} \mathrm{Dr}\end{array}$ \\
\hline Anfängl. Volum & . 210,7 & 0,2645 & 25.3 & 50,99 \\
\hline+ Sauerstoff & 307,6 & 0,3614 & 25,6 & 101,60 \\
\hline Nach der Explosion & 253,3 & 0,3071 & 25,6 & 71,11 \\
\hline - Kohlensäure & 216,2 & 0,2957 & 25,3 & 58,51 \\
\hline+ Wasserstotf & 478,2 & 0,5515 & 25,8 & 241,00 \\
\hline Nach der Explosion & 392,0 & 0,4673 & 26,5 & 167,00 \\
\hline
\end{tabular}

Diefs ergiebt einen Stickstoffgehalt von 56,84 pC.; der weiteren Berechnung wurde jedoch eine mit der ersten sehr nahe übereinkommende Bestimmung zu Grunde gelegt, welche etwas weniger Stickstoff ergab und zuverlässiger schien.

In die im Absorptionsrohr zurückgebliebene Gasportion wurde nun eine mit rauchender Schwefelsäure getränkte Coakskugel zur Absorption des Elayls und Ditetryls eingeführt und 20 Stunden darin gelassen, darauf die gebildele scliweflige Säure und Kohlensäure durch eine Kalikugel entfernt. Die Ablesungen ergaben :

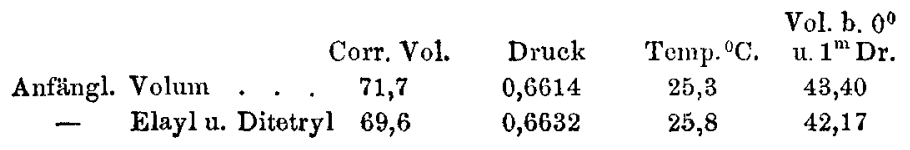


208 Lunge, Zusammensetzung des Gases im dunkelen Kegel

Daraus ergiebt sich in 100 Theilen :

$$
\begin{aligned}
& \begin{array}{lll|l}
\text { Elayl * * * } & \text { - } \\
\text { Ditetryl } & \text {. } & \text {. }
\end{array} \\
& \text { Kohlensïiure . . } 0,00 \\
& \text { Sauerstoff . . 14,28 } \\
& \text { Rest . . . } 83,29
\end{aligned}
$$

Der noch übrige Rückstand wurde wieder in das grofse

\begin{tabular}{|c|c|c|c|c|c|}
\hline Anfängl. Volum & . & . 225,5 & 0,2753 & 27,6 & 56,39 \\
\hline$+\quad$ Sauerstoff & . & 350,7 & 0,4003 & $27, \check{5}$ & 127,60 \\
\hline Nach der Explosion & - & 302,1 & 0,3530 & 27,1 & 97,01 \\
\hline - Kohlensäure . & - & 271,3 & 0,3498 & 27,0 & 86,38 \\
\hline + Wasserstoff & . & 532,8 & 0,6007 & 25,3 & 292,60 \\
\hline Nach der Explosion & . & 363,8 & 0,4411 & 26,0 & 146,50 \\
\hline
\end{tabular}
Eudiometer übergefüllt und ganz wie bei der ersten eudiometrischen Analyse behandelt. Die Ablesungen waren :

$$
\begin{array}{llll} 
& & \text { Vol. b. } 0^{0} \\
\text { Corr. Vol. Druck Temp. }{ }^{\circ} \mathrm{C} . & \text { u. } 1^{\mathrm{m}} \text { Dr. }
\end{array}
$$

Wenn man den Stickstoff hieraus berechnet, so ergiebt er $\operatorname{sich}=37,68$, oder, auf 83,29 Theile berechnet, $=55,65$; dieser Werth wurde, wie oben bemerkt, zu Grunde gelegt. Dann bleiben noch 18,71 (A) als Gesammtrolumen des Wasserstoffes, Kohlenoxyds und Grubengases (Schwefelwasserstoff enthält das Heidelberger Leuchtgas nicht). Ferner folgt aus diesen Ablesungen für die bei der Verbrennung gebildete Kohlensäure der Werth 10,63 (B); für die Contraction 30,59 (C). Bezeichnet man nun die in 18,71 Theilen enthaltene Menge Wasserstoff mit $x$, Kohlenoxyd mit $y$ und Grubengas mit $z$, so ergeben sich folgende Gleichungen :

$$
\begin{gathered}
x+y+z=A \\
y+z=B \\
11 / 2 x+1 / 2+2 z=C \\
x=A-B \\
y=A+\frac{B}{3}-2 \frac{C}{3} \\
z=\frac{2 B}{3}+2 \frac{C}{3}-A \\
x=8,08 \\
y=1,85 \\
z=8,78
\end{gathered}
$$


oder in 83,29 Theilen :

$$
\begin{aligned}
& x=11,93 \\
& y=2,73 \\
& z=12,97
\end{aligned}
$$

Wendet man diese Resultate auf die erstc cudiometrische Analyse an, so erhält man nach den Formeln in Bunsen's gasometrischen Methoden (S. 117) auf 2,43 des Gemenges von Elayl und Diletryl :

$$
\begin{aligned}
& \text { Elayl . . } 1,67 \\
& \text { Ditetryl . . 0,76 }
\end{aligned}
$$

Die Zusammensetzung des untersuchten Gasgemenges war also folgende :

$$
\begin{aligned}
& \text { Kohlensture . . } 0,00 \\
& \text { Sauerstoff . . . 14,28 } \\
& \text { Elayl . . . . } 1,67 \\
& \text { Ditetryl . . . } 0,76 \\
& \text { Kohlenoxyd . . . } \quad 2,73 \\
& \text { Wasserstoff . . 11,94 } \\
& \text { Grubengas . . 12,97 } \\
& \text { Stickstoff } \cdots \cdot \frac{55,65}{100,00}
\end{aligned}
$$

Durch viele früher ausgeführte Analysen des Heidelberger Leuchtgases, auch durch solche, welche gleichzeitig mit meiner Arbeit angestellt wurden, ist es erwiesen, dafs dasselbe kein Sauerstoffgas enthält; man darf daher den ganzen gefundenen Sauerstoffgehalt der atmosphärischen Luft des Gemenges zuschreiben, welche sich danach auf $68,13 \mathrm{pC}$. berechnet, wovon $53,85 \mathrm{pC}$. auf den Stickstoff kommen; es bleiben demnach 31,78 pC. Gas von folgender Zusammensetzung :

$$
\begin{aligned}
& \text { Kohlensäure . . . } 0,00 \\
& \text { Saucrstoff . . . } 0,00 \\
& \text { Elayl . . . . } 5,24 \\
& \text { Ditetryl . . . } 2,38 \\
& \text { Kohlenoxyd . . . } 8,58 \\
& \text { Wasserstoff . . } 37,46 \\
& \text { Grubengas . . . 40,70 } \\
& \text { Stickstoff . . . . 5,64 }
\end{aligned}
$$


Diese Zusammensetzung stimmt mit früheren Analysen des Heidelberger Leuchtgases, z. B. den in Bunsen's und Roscoe's photochemischen Untersuchungen angeführten von La n do lt (Pogg. Ann. C, 84), ganz gut überein.

Es läfst sich nun berechnen, wieviel Sauerstoff (resp. Luft) noch von aufsen der Flamme zur vollständigen Verbrennung zugeführt werden mufs. Nämlich :

\begin{tabular}{|c|c|c|c|c|c|c|}
\hline 1,67 & Vol & Elayl & wchen & 5,01 & Tol & Sauerstoff \\
\hline 0,76 & $n$ & Ditetryl & $n$ & 4,56 & $n$ & n \\
\hline 2,73 & $n$ & Kohlenoxyd & $n$ & 1,37 & $n$ & $n$ \\
\hline 11,94 & n & Wasserstoff & $n$ & 5,97 & $n$ & $n$ \\
\hline 97 & $n$ & Grubengas & $n$ & 25,94 & " & $n$ \\
\hline & & & & 42,85 & & \\
\hline
\end{tabular}

100 Theile des Gasgemenges brauchen daher zur vollständigen Verbrennung noch 42,85 $-14,28=28,57$ Theile Sauerstoff, welche 136,30 Theilen Luft entsprechen; es stellt sich also das auffallende Resultat heraus, dafs bei der Flamme des untersuchten Brenners dem Gase von innen fast ganz genau $1 / 3$ des Sauerstoffs (resp. der Luft) zugeführt wird, welcher zur Verbrennung desselben erforderlich ist.

Ich habe schliefslich noch nach dem Verfahren in Bunsen's gasometrischen Methoden (S. $247 \mathrm{ff}$.) Verbrennungswärme und Verbrennungstemperatur für den vorliegenden Fall berechnet. Es ergiebt sich danach, wenn $g$ das Gewicht jedes Gases, w die Verbrennungswärmen der Gemengtheile, $W$ die Verbrennungswärme des ganzen Gasgemenges bezeichnet :

\begin{tabular}{|c|c|c|c|c|c|}
\hline & & Vor der & erbrer & 19 : & \\
\hline Samerstof: & & $\begin{array}{l}\text { Vol. } \\
42.85\end{array}$ & $\underset{6, \mathbf{1}}{\mathbf{g}}$ & $\left.w^{*}\right)_{0}$ & $\mathrm{wg}_{0}$ \\
\hline Elayl. & . & 1.67 & 0,209 & 11640 & 2433,0 \\
\hline Ditetryl. & . & 0,76 & 0,190 & 11529 & 2191,0 \\
\hline Kohlenoxyd & & 2,73 & 0,342 & 2403 & 821,8 \\
\hline Wasserstoff & & 11,94 & 0,107 & 34462 & 3687,4 \\
\hline Grubengas & • & 12,97 & 0,928 & 13063 & 12123,0 \\
\hline Stickstoff & . & - 163,38 & 20,530 & 0 & 0 \\
\hline & & & 28,434 & $\Sigma g w$ & 21256,2 \\
\hline
\end{tabular}

- Nach Favre und Silbermann. 


$$
\mathrm{W}=\frac{\Sigma \mathrm{gw}}{\mathrm{gg}}=747,3 \text {. }
$$

Nach der Verbrennung :

(wenn $p$ das Gewicht, s die specifische Wärme der einzelnen Gase bezeichnet)

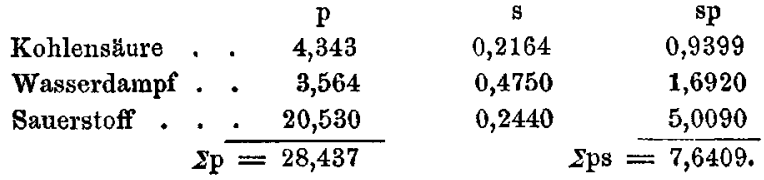

$$
\begin{aligned}
& \mathrm{s}=\frac{\Sigma \mathrm{ps}}{\Sigma \mathrm{p}}=0,26869 \text {. }
\end{aligned}
$$

Die Verbrennungstemperatur ist also $\frac{\mathrm{W}}{\mathrm{S}}=2781^{\circ} \mathrm{C}$. Heidelberg, Juli 1859.

\section{Ueber einige Selenmetalle; von G. Little*).}

Für die Darstellung der folgenden Selenmetalle wurde im Allgemeinen von der Voraussetzung ausgegangen, dals sie analoge Zusammensetzung, wie die Schwefelverbindungen derselben Metalle, haben möchten.

Selennickel. - In eine Röhre von schwer schmelzbarem Glas wurden $5 \mathrm{Grm}$. Selen gebracht, davor 2,25 Grm. Nickel in dünner Schichte; das Metall wurde zum Rothglühen erhitzt, dann das Selen in Dampf verwandelt. Bei dem Zutritt des Selendampfes zu dem glühenden Nickel trat unter Feuer-

*) Im Auszug aus dessen Inaugural-Dissertation : On selenium and some of the metallic seleniets; Göttingen 1859 . 